\title{
Simultaneous measurement of temperature and relative humidity using a dual-wavelength erbium doped fiber ring laser sensor
}

\author{
Mateusz Mądry, Lourdes Alwis, Leonardo Binetti, Łukasz Pajewski, Elżbieta Bereś-Pawlik, Senior \\ Member, IEEE
}

\begin{abstract}
A fiber ring laser sensor setup utilizing FBGs (Fiber Bragg Gratings) for simultaneous measurement of ambient temperature and relative humidity $(\mathrm{RH})$ is presented. Two FBGs are incorporated as tunable filters for a dual-wavelength laser emission, where one FBG was coated with Polyimide (PI) in order to achieve sensitivity to RH changes, while the other bare FBG was used for temperature sensing. An increase in RH would induce a strain on the grating, which results in a variation in the resonance wavelength of the PI-coated FBG. This causes a shift in the laser emission wavelength. Being insensitive to $R H$ changes, the bare FBG was employed to measure temperature. The dual-wavelength fiber ring laser sensor created thus allows to determine simultaneous measurement of RH and temperature. The RH sensitivities observed by the PI coated FBG to RH and temperature are $3.6 \mathrm{pm} / \% \mathrm{RH}$ and $12.15 \mathrm{pm} /{ }^{\circ} \mathrm{C}$ respectively. The temperature sensitivity of the bare FBG was observed to be 9.6 $\mathrm{pm} /{ }^{\circ} \mathrm{C}$. The main advantage of the proposed setup is an optical signal to noise ratio (OSNR) higher than $55 \mathrm{~dB}$ and a $3 \mathrm{~dB}$ bandwidth less than $0.02 \mathrm{~nm}$, which points out efficient capabilities for both precise sensing and remote detection applications.
\end{abstract}

Index Terms - fiber ring laser, fiber Bragg gratings, humidity sensor, temperature sensor

\section{INTRODUCTION}

$\mathrm{R}_{\mathrm{r}}^{\mathrm{e}}$ elative humidity (RH) is a crucial environmental parameter, which needs to be controlled in many fields of engineering, for instance agriculture, food processing, metrology or pharmacy [1]. Compared to the conventional techniques such as electrical monitoring devices, optical fiber sensors have been intensely developed due to their many advantages, i.e. immunity to electromagnetic interferences, compact-size, high sensitivity, long-term durability and resistance to harsh environment [2]. Thus, different types of optical fiber humidity sensors have been reported so far, which could be classified into several groups depending on their physical structure and method of interrogation, i.e. optical absorption sensors [3, 4], long-period gratings (LPGs) [5,6],

This work was co-financed by the Designated Subsidy for Young Scientists, no. 0402/0159/18 and statutory funds of Telecommunications and Teleinformatics Department, Wroclaw University of Science and Technology, no. 0401/0023/18.

M. Mądry and Ł. Pajewski are with the Telecommunications and Teleinformatics Department, Faculty of Electronics, Wroclaw University of fiber Bragg gratings (FBGs) [7,8], interferometers (Fabry-Pérot [9,10], Sagnac [11,12], resonators [13], lossy mode resonances [14] or modal interferometers $[15,16])$. The sensing regions were often coated by various materials, for instance, polymethyl methacrylate (PMMA), polyvinyl acetate (PVA) or polyimide (PI), to obtain sensitivity to humidity $[17,18]$.

One strong candidate for sensing applications is the use of a fiber ring laser (FRL) in view of its advantages, i.e. a high optical signal to noise ratio (OSNR), high intensity and low spectral width [19]. In general, the FRL consists of a ring laser tuned by appropriate photonic structures, which have an influence on its laser properties. When the sensing structure is altered, the laser emission spectrum changes its properties, e.g. experiences a wavelength shift. The high OSNR in FRLs enables remote detection of a particular target measurement at long distance and improves the detection accuracy. Due to high OSNR, low spectral width and high intensity FRLs are of great research interest [20-30]. Different structures had been proposed within FRL setups for sensing applications, i.e. interferometers, i.e. Mach-Zehnder [20,21], Fabry-Pérot [22,23], Sagnac [24,25], and FBGs [26,27]. Shi et al. presented a FRL sensor combined with a Fabry-Pérot interferometer (FPI) [28], where an Agarose film was deposited on the fiber to obtain sensitivity to humidity, yielding a sensitivity of $0.202 \mathrm{~dB} / \% \mathrm{RH}$. The SNR of the said setup was $30 \mathrm{~dB}$ and the $3-\mathrm{dB}$ bandwidth less than $0.05 \mathrm{~nm}$. In contrast, the sensing scheme proposed in this paper is based on a dual-wavelength FRL setup in order to measure $\mathrm{RH}$ and temperature changes simultaneously by exploiting FBGs as optical filters. The proposed setup yielded an OSNR higher than $55 \mathrm{~dB}$, which allows the enhancement of the potential to remotely monitor humidity and temperature as the dynamic range is much increased compared to just utilizing FBGs. For example, if a standard broadband light source is used with the FBGs, i.e. superluminescent light emitting diode (SLED), the OSNR would be much lower in comparison to the proposed FRL setup. The FBGs, used within FRL, induces a narrower spectral width that enhances sensing and accuracy

Science and Technology, Wyb. Wyspiańskiego 27, 52-370 Wrocław, Poland (e-mail: mateusz.madry@pwr.edu.pl).

L. Alwis and L. Binetti are with the School of Engineering and The Built Environment, Edinburgh Napier University, 10 Colinton Road, Edinburgh, Scotland, UK. 
capabilities, i.e. laser emission wavelength shift could be clearly determined due to its low spectral width, in contrast to the setup which uses only a broadband light source with much broader spectral width. FRLs that utilize FBGs has already been presented in the literature. For example, Bui et al. used two FBGs in a FRL setup in order to measure organic solvents, i.e. ethanol and methanol in gasoline and nitrate, in water [29]. Another example is the work carried out by Shao et al. [30], who presented a refractive index FRL sensor setup based on two FBGs, obtaining an OSNR higher than $60 \mathrm{~dB}$ and a spectral width lower than $0.02 \mathrm{~nm}$. Shao et al. used cladding-etched FBGs, which was thus made sensitive to external refractive index changes, while using another FBG as a reference laser emission. In both these work, the temperature influence was not taken into consideration $[29,30]$.

The work presented here targets the measurement of $\mathrm{RH}$, while taking the temperature fluctuations into account. The setup is also suitable for remote monitoring because of high OSNR following the paper presented by Shi et al. [25]. The experimental results reveal an OSNR higher than $55 \mathrm{~dB}$ and a spectral width much narrower than that of typical highreflectivity FBGs, i.e. $\leq 0.02 \mathrm{~nm}$, which indicates its strong suitability to be deployed for sensing. Hence, a novel measurement of both RH and ambient temperature utilizing a dual-wavelength FRL setup based on bare and PI-coated FBGs, is demonstrated through the work presented herewith. The paper demonstrates the concept and experimental results of the FRL sensor for RH and temperature monitoring. The scheme thus proposed for the measurement of RH is first of its kind to the best of the authors' knowledge.

\section{FBG SENSING MECHANISM}

A FBG is a structure made by modulating the refractive index of the core of a fiber. When illuminated by broadband light, it reflects the wavelength which fulfill the Bragg condition [32]. This wavelength, i.e. the Bragg wavelength, is directly related to the effective refractive index of the core $\left(n_{\text {eff }}\right)$ and the grating period $(\Lambda)$, which is given by:

$$
\lambda=2 n_{e f f} \Lambda
$$

As can be seen from equation 1 , any variation in either the effective refractive index of the core or the grating period, i.e. resulting from an elongation of the fibre, will induce a resonance wavelength shift. Therefore, both the ambient temperature and strain affect the Bragg wavelength, which is described by the following equation [32]:

$$
\frac{\Delta \lambda_{B}}{\lambda_{B}}=\left(1-P_{e}\right) \varepsilon+\left[\left(1-P_{e}\right) \alpha+\xi\right] \Delta T
$$

where $\varepsilon$ and $\Delta T$ is applied strain and change of temperature respectively, $P_{e}$ is a photoelastic constant of the fiber, $\alpha$ refers to thermal expansion coefficient of the fiber and $\xi$ corresponds to the thermos-optic coefficient. This equation determines the strain and temperature effects on the FBG. If the influence of humidity is considered, this equation should be changed to the following form [32]:

$$
\frac{\Delta \lambda_{B}}{\lambda_{B}}=\left(1-P_{e}\right) \alpha_{R H} \Delta R H+\left[\left(1-P_{e}\right) \alpha_{T}+\xi\right] \Delta T
$$

In this equation, $\alpha_{R H}$ is the expansion coefficient due to moisture and $\alpha_{T}$ is the thermal expansion coefficient, which depicts the shift in the Bragg wavelength towards longer or shorter wavelengths due to applied strain resulting from moisture variations. The same is true for thermal expansion and thermooptic effects. Using these phenomena, the proposed laser emission wavelength could be tuned to follow the changes in RH. One FBG was coated with polyimide (PI-coated FBG) in order to provide RH sensitivity. PI is a suitable coating material due to its reproducibility and linear response to moisture changes [31]. The PI-coated FBG affected by moisture absorption or desorption causes shifts in its resonance wavelength and consecutively in the laser emission wavelength. Being insensitive to RH changes, the bare FBG could shift only due to ambient temperature.

The tracking of laser peak emission wavelengths allow the determination of both temperature and $\mathrm{RH}$ changes according to the following matrix equation:

$$
\left[\begin{array}{c}
\Delta \lambda_{P I-F B G} \\
\Delta \lambda_{F B G}
\end{array}\right]=\left[\begin{array}{cc}
S_{T, P I-F B G} & S_{H, P I-F B G} \\
S_{T, F B G} & 0
\end{array}\right]\left[\begin{array}{c}
\Delta T \\
\Delta R H
\end{array}\right]
$$

where $S_{H, P I-F B G}$ is the humidity sensitivity of the PI-coated FBG. $S_{T, P I-F B G}$ and $S_{T, F B G}$ refer to temperature sensitivities of the PIcoated FBG and the bare FBG, respectively. In order to determine temperature and humidity, the matrix above (4) should also include initial values of both the FBG-based laser emission wavelengths, i.e. $\lambda_{P I-F B G, 0}$ refers to the PI-coated laser and $\lambda_{\text {Bare-FBG, },}$, refers to the bare FBG laser, respectively. This results in:

$$
\left[\begin{array}{c}
T \\
R H
\end{array}\right]=\left[\begin{array}{cc}
S_{T, P I-F B G} & S_{H, P I-F B G} \\
S_{T, F B G} & 0
\end{array}\right]^{-1}\left[\begin{array}{c}
\lambda_{P I-F B G} \\
\lambda_{B a r e-F B G}
\end{array}\right]-\left[\begin{array}{c}
\lambda_{P I-F B G, 0} \\
\lambda_{B a r e-F B G, 0}
\end{array}\right]
$$

\section{MATERIALS AND METHODS}

The FBGs were inscribed using a mechanism that is described in detail elsewhere [33]. A PI solution was used to coat one of the FBGs to make it sensitive to humidity changes. Prior to the coating of the PI layer, the FBG was annealed at $185^{\circ} \mathrm{C}$ for 3 hours to ensure stability. The PI material used was supplied by HD Microsystems in liquid form, having a refractive index of 1.70 . The grating was firstly covered by $3-$ aminopropyltriethoxysilane (3-APTS) solution $(0.01 \%)$ to achieve a strong bond, i.e. adequate bonding for the strain transfer to occur, between the fiber surface and the PI layer. The fiber containing the thus coated FBG was then placed in the oven for 15 minutes at a temperature of $130^{\circ} \mathrm{C}$. Afterwards, the FBG was dip-coated at a velocity of $13 \mathrm{~mm} / \mathrm{min}$ in the PI solution and it was then subjected to a temperature of $150{ }^{\circ} \mathrm{C}$ for a duration of 5 minutes. This process was repeated until several number of layers were deposited on the grating, providing an adequate coating. Finally, the coated grating was cured in the oven for approximately 1 hour at $180{ }^{\circ} \mathrm{C}$. The thickness of the coating was approximately $24 \mu \mathrm{m}$.

\section{EXPERIMENTAL SETUP}

The proposed fiber sensor setup consists of a single-mode pigtailed laser diode (BL976-SAG300, $\lambda=976 \mathrm{~nm}$, Output power: $300 \mathrm{~mW}$, Thorlabs), a WDM coupler $(980 / 1550 \mathrm{~nm})$, $60 \mathrm{~cm}$ length of polarization-maintaining erbium doped fiber as 
a gain medium (PM-EDF, Absorption coefficient: $55 \pm 5 \mathrm{~dB} / \mathrm{m}$ near $1530 \mathrm{~nm}$, MFD (at $1550 \mathrm{~nm}$ ): $8.8 \pm 1 \mu \mathrm{m}$, Birefringence: $3.5 \cdot 10^{-4}$, Nufern), a 3-port optical circulator (OC), a variable optical attenuator (VOA), the PI-coated and bare FBGs, a polarization controller (PC) and two other fiber couplers. The optical spectrum analyzer (OSA, Wavelength range: 600-1700

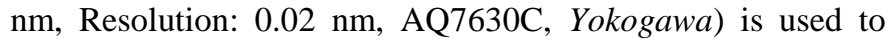
monitor the laser spectra. By adjustment of the VOA, the equalization of losses can be achieved, which allows to obtain dual-wavelength laser emission corresponding to the FBGs. The 3-dB coupler is used to split light into two fibers, each containing an FBG. In the meantime, another coupler (95:5) is used to extract $5 \%$ of propagating light directly to the OSA. The optical circulator ensures directional propagation of light within the proposed setup. The whole fiber ring laser setup scheme is presented in Fig. 1.

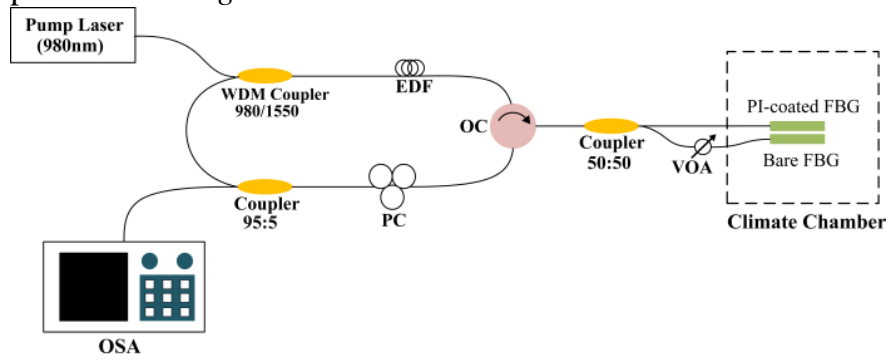

Fig. 1. The proposed fiber ring laser sensor setup based on FBGs for temperature and humidity measurement.

PM-EDF was used as a gain medium due to its mode hopping suppression over single-mode erbium doped fiber (SM-EDF) [34]. Laser emission wavelengths resulting from the PI-coated FBG and the bare FBG were $1542.32 \mathrm{~nm}$ and $1544.632 \mathrm{~nm}$ respectively (measured at $\mathrm{T}=20{ }^{\circ} \mathrm{C}$ and $\mathrm{RH}=40 \% \mathrm{RH}$ ). Laser linewidths did not exceed $0.015 \mathrm{~nm}$ (PI-coated FBG) and 0.02 $\mathrm{nm}$ for the bare FBG. Both the FBG spectra and the laser emission are presented in Fig. 2.

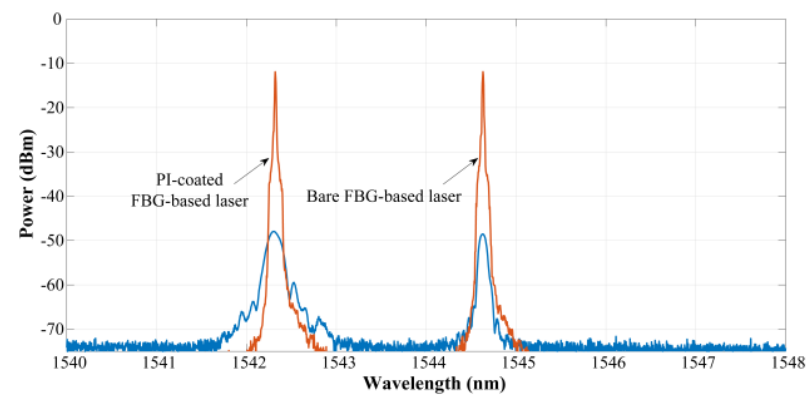

Fig. 2. The spectra from the FBGs and the proposed dual-wavelength fiber ring laser $\left(\mathrm{T}=20^{\circ} \mathrm{C}\right.$ and $\left.\mathrm{RH}=40 \% \mathrm{RH}\right)$.

\section{RESULTS}

\section{A. RH response}

Both FBGs were placed in a certificated climate chamber (KMF 115, Binder), which could be set to vary RH and temperature conditions. Moreover, the thickness of the PI coating has an influence on the Bragg wavelength shift. If the PI layer is thicker, the grating will be more strained, which causes a larger shift of Bragg wavelength, respectively $[32,36]$. However, even though the sensitivity will be higher, the response time will be longer as the polymer layer will take more time to expand a higher mass than for a relatively thinner coating mass. The thickness achieved was observed to be of adequate sensitivity for the purpose of the work presented here.

The proposed sensor setup was investigated for the range of $20-80 \% \mathrm{RH}$ in steps of $20 \% \mathrm{RH}$, while maintaining a constant temperature of $20^{\circ} \mathrm{C}$. All of the spectral measurements for each $\mathrm{RH}$ were conducted after one hour of stabilization and data was recorded using the OSA. The measurements for each value of $\mathrm{RH}$ were repeated four times and the average laser emission wavelength was computed as well as its deviation. The laser emission spectra for different RH values are presented in Fig. 3(a), whereas the shift of PI-coated FBG-based laser wavelength as a function of $\mathrm{RH}$ is illustrated in Fig. 3(b).

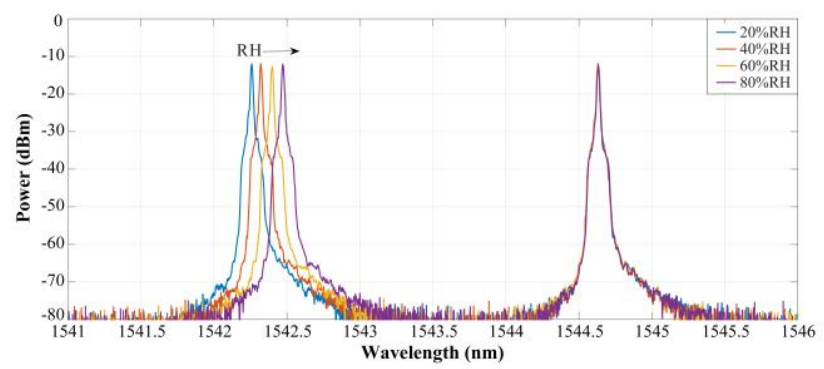

(a)



(b)

Fig. 3. Laser emission spectra due to RH changes (a) and the resulting wavelength shift of the laser (b).

As expected, the PI-coated FBG-based laser spectrum experienced a red-shift as RH increased from 20 to $80 \% \mathrm{RH}$. This is due to the strain induced on the grating from the swelling of the PI layer as it absorbs moisture vapor [35]. A linear relationship between laser wavelength and $\mathrm{RH}$ was obtained $\left(\mathrm{R}^{2}=0.99\right)$ with a $\mathrm{RH}$ sensitivity of $3.6 \mathrm{pm} / \% \mathrm{RH}($ Fig. 3(b)). The observed linear response is consistent with literature [31, $36,37]$. In the meantime, no spectral shift from the bare FBG was observed during the RH changes, as expected.

\section{B. Temperature response}

The temperature sensitivity was investigated for both laser emission peaks resulting from the FBGs. To do so, the ambient temperature was changed from $10-40{ }^{\circ} \mathrm{C}$ in steps of $10{ }^{\circ} \mathrm{C}$, whereas RH was kept constant over the whole measurement (40 $\% \mathrm{RH})$. The measurements for each value of temperature were repeated four times and the average laser emission wavelength was computed as well as its variation. The measurement results are presented in Fig. 4 for both the PI-coated FBG (Fig. 4(a)) and the bare FBG (Fig. (4b)), respectively. 


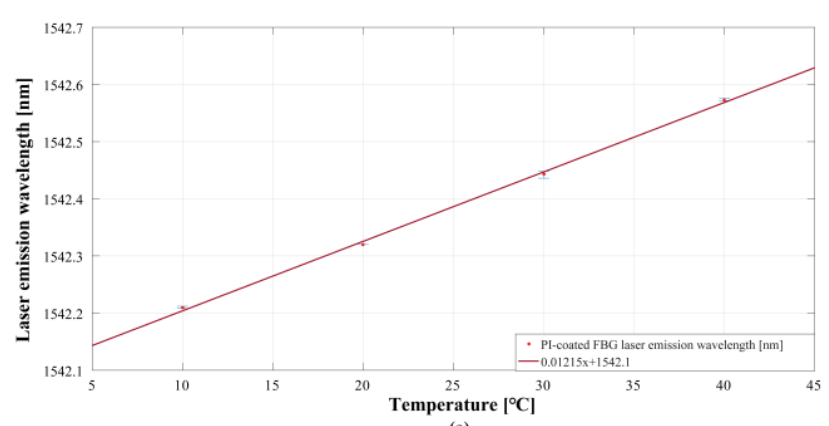

(a)

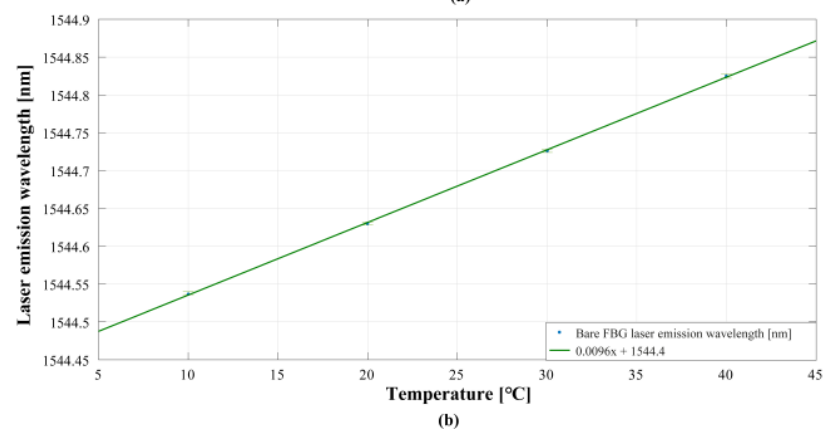

Fig. 4. The temperature response of the PI-coated FBG (a) and bare FBG laser (b).

As can be seen from Fig. 4, a linear response $\left(\mathrm{R}^{2}=0.999\right)$ to temperature is observed for both the laser emission wavelengths. Both the bare FBG and PI-coated FBGs responds to temperature linearly, which can already be confirmed from the literature $[38,39]$. The temperature sensitivities of the PIcoated FBG and the bare $\mathrm{FBG}$ lasers were $12.15 \mathrm{pm} /{ }^{\circ} \mathrm{C}$ and 9.6 $\mathrm{pm} /{ }^{\circ} \mathrm{C}$ respectively. Due to the occurrence of mode hopping in laser setups, multiple measurements were performed. Maximal observed wavelength variation during the repeated measurements (of both RH and temperature FRL response) was $12 \mathrm{pm}$, which points out adequate stability of the proposed laser sensor.

\section{DISCUSSION}

According to the measurement data, the matrix sensitivity equations can be written for the proposed dual-wavelength laser sensor. The RH sensitivity is $3.6 \mathrm{pm} / \% \mathrm{RH}$, whereas temperature sensitivity is $9.6 \mathrm{pm} /{ }^{\circ} \mathrm{C}$ for the bare $\mathrm{FBG}$-based laser emission and $12.15 \mathrm{pm} /{ }^{\circ} \mathrm{C}$ for the PI-coated $\mathrm{FBG}$ laser emission. The matrix sensitivity equation could thus be presented as follows:

$$
\left[\begin{array}{c}
\lambda_{P I-F B G} \\
\lambda_{\text {Bare-FBG }}
\end{array}\right]=\left[\begin{array}{cc}
0.01215 & 0.0036 \\
0.096 & 0
\end{array}\right]\left[\begin{array}{c}
T \\
R H
\end{array}\right]+\left[\begin{array}{l}
1541.9385 \\
1544.4395
\end{array}\right]
$$

To determine temperature and $\mathrm{RH}$ values, the matrix above (6) is converted into the following form:

$$
\left[\begin{array}{c}
T \\
R H
\end{array}\right]=\left[\begin{array}{cc}
0 & 104.2 \\
277.8 & -351.6
\end{array}\right]\left[\begin{array}{c}
\lambda_{P I-F B G} \\
\lambda_{\text {Bare-FBG }}
\end{array}\right]-\left[\begin{array}{c}
1541.9385 \\
1544.4395
\end{array}\right]
$$

Evaluation of the proposed setup is an important aspect, thus the figure of merit (FOM) for a sensor is introduced according to the following equation [30]:

$$
F O M=\frac{S_{\lambda}}{F W H M}
$$

The performance of the sensor is directly related to its sensitivity $\left(\mathrm{S}_{\lambda}\right)$ and spectral bandwidth (FWHM). Assuming similar sensitivity to $\mathrm{RH}$ for the PI-coated FBG ( 4 pm/\% RH), which is comparable with the results presented in the literature $[32,36]$, the FWHM of the laser is lower $(\leq 0.02 \mathrm{~nm})$ than the bandwidth of the FBG $(\sim 0.15 \mathrm{~nm})$ which is illuminated by just a broadband light source, which increases the FOM (Eq. 7) significantly. Apart from the advantage of a narrow spectral width, the proposed setup exhibits OSNR higher than $55 \mathrm{~dB}$, which allow for remote monitoring of the changes in ambient temperature and RH. Thus the proposed setup is highly beneficial for remote monitoring target applications.

Additionally, due to observed temperature cross-sensitivity, validation of the proposed sensor was performed by means of measurements of laser wavelengths for different temperature and humidity values [40]. Although the bare FBG and PI-coated FBG are approximately placed in the same place, an error could be observed. As it is seen from the Fig. 4 the temperature sensitivity is significant, thus the necessary validation should be conducted. The different temperature and humidity values were chosen to assess the calculated model. Temperature was changed from $10{ }^{\circ} \mathrm{C}$ to $40{ }^{\circ} \mathrm{C}$, whereas humidity was varied over a range of 20 to $80 \% \mathrm{RH}$. Both applied and measured temperature and humidity values are presented in the Fig. 5.

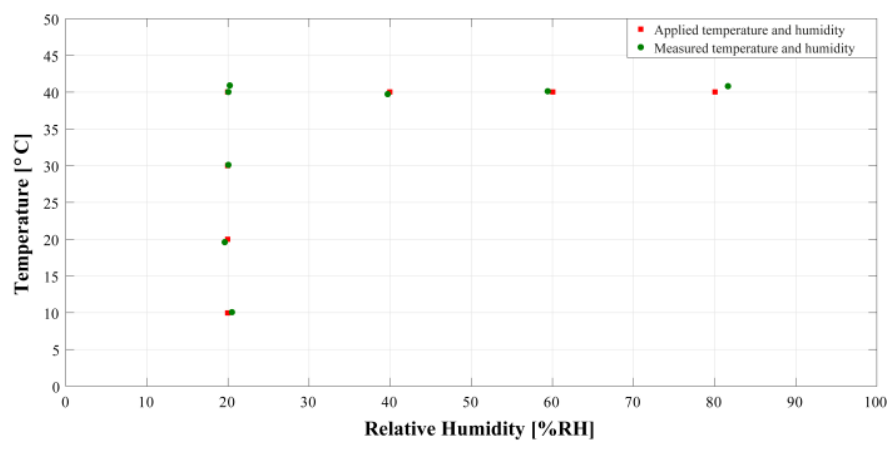

Fig. 5. The validation of proposed experimental setup by comparison of applied and measured temperature and humidity values.

The sensor assessment shows good sensing capabilities. Maximal temperature error between applied and measured values did not exceed $0.48{ }^{\circ} \mathrm{C}$. In the meantime, measured humidity differed from the applied by $1.64 \% \mathrm{RH}$ in the worst case during the sensor evaluation. This validation proves good accuracy of proposed sensor. Work is currently ongoing to multiplex a number of FBGs within a FRL setup in order to create sensor arrays. In addition, the sensitivity to humidity is also planned to be enhanced in the future through further characterization of the coating material properties.

\section{CONCLUSIONS}

A dual-wavelength FRL sensor setup based on two FBGs for $\mathrm{RH}$ and ambient temperature measurement is proposed and experimentally proven. FBGs were used in order to create the sensing elements. A PI-coated FBG was utilized as the RH sensing element while a bare FBG was used for ambient temperature measurement. Achieved RH sensitivity was 3.6 $\mathrm{pm} / \% \mathrm{RH}$ and temperature sensitivities were $9.6 \mathrm{pm} /{ }^{\circ} \mathrm{C}$ and $12.15 \mathrm{pm} /{ }^{\circ} \mathrm{C}$ corresponding to the bare FBG and the PI-coated FBG respectively. Experimental results revealed good laser performance, i.e. OSNR higher than $55 \mathrm{~dB}$ and $3 \mathrm{~dB}$ bandwidth less than $0.02 \mathrm{~nm}$. Additional validation of the proposed sensor pointed out that the maximal experimental temperature and 
humidity errors were $0.48{ }^{\circ} \mathrm{C}$ and $1.64 \% \mathrm{RH}$, respectively. Moreover, due to high OSNR, the proposed sensor scheme is highly suitable for remote monitoring of $\mathrm{RH}$ and ambient temperature. The presented FRL setup could be enhanced by FBGs in order to create a fiber sensor network.

\section{REFERENCES}

[1] L. Alwis, T. Sun and K. T. V. Grattan, "Optical fibre-based sensor technology for humidity and moisture measurement: Review of recent progress," Measurement, vol. 46, no. 10, pp. 4052-4074, Dec. 2013.

[2] J. Ascorbe, J. M. Corres, F. J. Arregui, and I. R. Matias, "Recent Developments in Fiber Optics Humidity Sensors," Sensors, vol. 17, no. 4, pp. 893, Apr. 2017.

[3] Y. Xiao, J. Zhang, X. Cai, S. Tan, J. Yu, H. Lu, Y. Luo, G. Liao, S. Li, J. Tang and Z. Chen, "Reduced graphene oxide for fiber-optic humidity sensing," Opt. Express, vol. 22, no. 25, pp. 31555-31567, Dec. 2014.

[4] R. Aneesh and S. K. Khijwania, "Zinc oxide nanoparticle based optical fiber humidity sensor having linear response throughout a large dynamic range," Appl. Opt., vol. 50, no. 27, pp. 5310-5314, Sep. 2011.

[5] Y. Liu, L. Wang, M. Zhang, D. Tu, X. Mao and Y. Liao, "Long-Period Grating Relative Humidity Sensor with Hydrogel Coating," IEEE Photonics Technol. Lett., vol. 19, no. 12, pp. 880-882, June 2007.

[6] K. M. Tan, C. M. Tay, S. C. Tjin, C. C. Chan and H. Rahardjo, "High relative humidity measurements using gelatin coated long-period grating sensors," Sens. Actuators B: Chem., vol. 110, no. 2, pp. 335-341, Oct. 2005.

[7] S. F. H. Correia, P. Antunes, E. Pecoraro, P. P. Lima, H. Varum, L. D. Carlos, R. A. S. Ferreira and P. S. André, "Optical Fiber Relative Humidity Sensor Based on a FBG with a Di-Ureasil Coating," Sensors, vol. 12, no. 7, pp. 8847-8860, June 2012.

[8] G. Berruti, M. Consales, M. Giordano, L. Sansone, P. Petagna, S. Buontempo, G. Breglio and A. Cusano, "Radiation hard humidity sensors for high energy physics applications using polyimide-coated fiber Bragg gratings sensors," Sens. Actuators B: Chem., vol. 177, pp. 94-102, Feb. 2013.

[9] C. Huang, W. Xie, M. Yang, J. Dai, B. Zhang and S. Member, "Optical Fiber Fabry-Pérot Humidity Sensor Based on Porous $\mathrm{Al}_{2} \mathrm{O}_{3}$ Film," IEEE Photonics Technol. Lett., vol. 27, no. 20, pp. 2127-2130, Oct. 2015.

[10] J. S. Santos, I. M. Raimundo, C. M. B. Cordeiro, C. R. Biazoli, C.A.J. Gouveia and P. A. S. Jorge, "Characterisation of a Nafion film by optical fibre Fabry-Perot interferometry for humidity sensing," Sens. Actuators B: Chem., vol. 196, pp. 99-105, June 2014.

[11] J. Wang, H. Liang, X. Dong and Y. Jin, "A Temperature-Insensitive Relative Humidity Sensor by using Polarization Maintaining Fiber-Based Sagnac Interferometer," Microw. Opt. Technol. Lett., vol. 55, no. 10, pp. 2305-2307, Oct. 2013

[12] L.-P. Sun, J. Li, L. Jin, Y. Ran and B.-O. Guan, "High-birefringence microfiber Sagnac interferometer based humidity sensor," Sens. Actuators B: Chem., vol. 231, pp. 696-700, Aug. 2016.

[13] Y. Zheng, X. Dong, C. Zhao, Y. Li, L. Shao and S. Jin, "Relative Humidity Sensor Based on Microfiber Loop Resonator," Adv. Mater. Sci. Eng., vol. 2013, 815930, pp. 1-4, 2013.

[14] P. Sanchez, C. R. Zamarreño, M. Hernaez, I. del Villar, I. R. Matias, F. J. Arregui, "Humidity sensor fabricated by deposition of $\mathrm{SnO}_{2}$ layers onto optical fibers," in Proc. of Fifth European Workshop on Optical Fibre Sensors, 87940C, Krakow, Poland, May 2013.

[15] J. Mathew, Y. Semenova and G. Farrell, "Experimental demonstration of a high-sensitivity humidity sensor based on an Agarose-coated transmission-type photonic crystal fiber interferometer," Appl. Opt., vol. 52, no. 16, pp. 3884-3890, June 2013.

[16] D. Lopez-Torres, C. Elosua, J. Villatoro, J. Zubia, M. Rothhardt, K. Schuster and F. J. Arregui, "Photonic crystal fiber interferometer coated with a PAH/PAA nanolayer as humidity sensor," Sens. Actuators B: Chem., vol. 242, pp. 1065-1072, Apr. 2017.
[17] G. Woyessa, K. Nielsen, A. Stefani, C. Markos and O. Bang, "Temperature insensitive hysteresis free highly sensitive polymer optical fiber Bragg grating humidity sensor," Opt. Express, vol. 24, no. 2, pp. 1206-1213, Jan. 2016.

[18] T. Li, X. Dong,; C. C. Chan, K. Ni, S. Zhang and P. P. Shum, "Humidity Sensor with a PVA-Coated Photonic Crystal Fiber Interferometer, " IEEE Sens. J., vol. 13, no. 6, pp. 2214-2216, June 2013.

[19] W.-G. Xie, Y.-N. Zhang, P.-Z. Wang and J.-Z. Wang, "Optical Fiber Sensors Based on Fiber Ring Laser Demodulation Technology," Sensors, vol. 18 , no. 2, pp. 505, Feb. 2018

[20] L. J. Liang, G. B. Ren, B. Yin, W. J. Peng, X. Liang and S. S. Jian, "Refractive index and temperature sensor based on fiber ring laser with STCS fiber structure," IEEE Photonics Technol. Lett., vol. 26, no. 21, pp. 2201-2204, Nov. 2014.

[21] R. Xing, Z. X. Wang, Y. X. Gao, Y. H. Qi and S. S. Jian, "RI ring laser sensor based on concatenating CLF and SMF with one core-offset joint," IEEE Photonics Technol. Lett., vol. 28, no. 11, pp. 1225-1228, June 2016.

[22] H. Zou, L. Ma, H. Xiong, Y. S. Zhang and Y. T. Li, "Fiber ring laser sensor based on Fabry-Perot cavity interferometer for temperature sensing," Laser Phys., vol. 28, no.1, 015102, Jan. 2018.

[23] J. Shi, D. Xu, W. Xu, Y. Wang, C. Yan, C. Zhang, D. Yan, Y. He, L. Tang, W. Zhang, T. Liu and J. Yao, "Humidity sensor based on FabryPerot interferometer and intracavity sensing of fiber laser," J. Lightwave Technol., vol. 35, no. 21, pp. 4789-4795, Nov. 2017.

[24] L. Wang, N. Fang, C. Wu, H. Qin and Z. Huang, "A fiber optic PD sensor using a balanced Sagnac interferometer and an EDFA-based DOP tunable fiber ring laser," Sensors, vol. 14, no. 5, pp. 8398-8422, May 2014.

[25] J. Shi, Y. Wang, D. Xu, H. Zhang, G. Su, L. Duan, C. Yan, D. Yan, S. Fu and J. Yao, "Temperature sensor based on fiber ring laser with Sagnac loop," IEEE Photonics Technol. Lett., vol. 28, no. 7, pp. 794-797, Apr. 2016.

[26] M. A. Gonzalez-Reyna, E. Alvarado-Mendez, J. M. Estudillo-Ayala, E. Vargas-Rodriguez, M. E. Sosa-Morales, J. M. Sierra-Hernandez, D. Jauregui-Vazquez and R. Rojas-Laguna, "Laser Temperature Sensor Based on a Fiber Bragg Grating," IEEE Photonics Technol. Lett., vol. 27, no. 11, pp. 1141-1144, June 2015.

[27] R. Yang, Z. Lianqing, Y. Zhang, X. Lou and M. Dong, "Erbium-doped fiber ring laser sensor for high temperature measurements based on a regenerated fiber Bragg grating," Fiber and Integrated Optics, vol. 36, no. 6, pp. 252-261, Dec. 2017.

[28] J. Shi, D. G. Xu, W. Xu, Y. Wang, C. Yan, C. Zhang, D. Yan,; Y. He, L. Tang, W. Zhang, T. Liu and J. Yao, "Humidity sensor based on FabryPerot interferometer and intracavity sensing of fiber laser," J. Lightwave Technol., vol. 35, no. 21, pp. 4789-4795, Nov. 2017.

[29] H. Bui, T. B. Pham, V. A. Nguyen, V. D. Pham, T. C. Do, T. V. Nguyen, T. H. C. Hoang, H. T. Le and V. H. Pham, "Novel method of dual fiber Bragg gratings integrated in fiber ring laser for biochemical sensors," Measurement Science and Technology, vol. 29, no. 5, 055105, May. 2018.

[30] L. Y. Shao, J. W. Liang, X. P. Zhang, W. Pan and L. S. Yan, "Highresolution refractive index sensing with dual-wavelength fiber laser," IEEE Sens. J., vol. 16, no. 23, pp. 8463-8467, Dec. 2016.

[31] L. Alwis, T. Sun, and K. T. V. Grattan, "Design and performance evaluation of polyvinyl alcohol/polyimide coated optical fibre gratingbased humidity sensors," Review of Scientific Instruments, vol. 84, no. 2, 025002, Feb. 2013.

[32] T. L. Yeo, Tong Sun, K. T. V. Grattan, D. Parry, R. Lade and B. D. Powell, "Polymer-coated fiber Bragg grating for relative humidity sensing," IEEE Sensors Journal, vol. 5, no. 5, pp. 1082-1089, Oct. 2005.

[33] L. S. M. Alwis, T. Sun and K. T.V. Grattan, "Fibre Grating-based Sensor Design for Humidity Measurement in Chemically Harsh Environment," Procedia Engineering, vol. 168, pp. 1317-1320, 2016.

[34] H. Zou, S. Lou, W. Su and B. Han, "A stable multi-wavelength PM-EDF laser based on a nonlinear amplifying loop mirror and a TCF comb filter," Laser Phys., vol. 24, no. 1, Jan. 2014. 
[35] Y. Lin, Y, Gong, Y, Wu, and H, Wu, "Polyimide-Coated Fiber Bragg Grating for Relative Humidity Sensing, Photonic Sensors," vol. 5, no. 1, pp. 60-66, Mar. 2015.

[36] A. J. Swanson, S. G. Raymond, S. Janssens, R. D. Breukers, M. D. H. Bhuiyan, J. W. Lovell-Smith and M. R. Waterland, "Investigation of polyimide coated fibre Bragg gratings for relative humidity sensing," Meas. Sci. Technol., vol. 26, no. 12, 125101, Dec. 2015.

[37] P. Kronenberg, Pramod K. Rastogi, P. Giaccari, and H. G. Limberger, "Relative humidity sensor with optical fiber Bragg gratings," Opt. Lett., vol. 27, no. 16, pp. 1385-1387, Aug. 2002.

[38] P. Lu, L. Men and Q. Chen, "Polymer-Coated Fiber Bragg Grating Sensors for Simultaneous Monitoring of Soluble Analytes and Temperature", IEEE Sensors Journal, vol. 9, no. 4, pp. 340 - 345, Apr. 2009.

[39] H. Meng, W. Shen, G. Zhang, C. Tan and X. Huang, "Fiber Bragg gratingbased fiber sensor for simultaneous measurement of refractive index and temperature," Sensors and Actuators B: Chemical, vol. 150, no. 1, pp. 226-229, Sep. 2010.

[40] C. A. R. Díaz et al., "Liquid Level Measurement Based on FBGEmbedded Diaphragms With Temperature Compensation," IEEE Sensors Journal, vol. 18, no. 1, pp. 193-200, Jan. 2018. 